\title{
Mobile Continuous Reading
}

Chen-Hsiang Yu

MIT CSAIL

32 Vassar St

Cambridge, MA 02139

chyu@mit.edu

\begin{abstract}
This research focuses on mobile continuous reading under frequent context switching while reading web pages. This paper presents the results of a user study with 10 users. Four conditions were investigated in the study: visual-reading, audio-listening, manual-

switching between visual and audio, and auto-switching between them. The results showed that auto-switching not only provides the easiest reading experience, but it also results in significantly fewer missteps while walking, compared with visual-reading.
\end{abstract}

\section{Keywords}

Mobile reading

\section{ACM Classification Keywords}

H.5.2 [Information Interfaces And Presentation]: User Interfaces - Interaction styles;

\section{General Terms}

Design, Measurement

\section{Introduction}

The mobile device is used with a short attention span and users are likely to switch activities while using the device [6]. Mobility raises another challenge which has not been adequately addressed in the past: how to support continuous mobile reading in the presence of frequent context switches, such as switching from sitting to walking, to driving, or to hands-free 
conditions. Different contexts impose different constraints. For example, driving may prevent the user from holding the device.

Since focusing on the display of a mobile phone may be difficult while walking or driving, Vadas et al. showed that audio is an acceptable modality for mobile comprehension of text [7]. Although audio has been used in screen readers for blind or visually impaired people[1][4][5], there has been no clear understanding how to combine the audio modality with visual presentation for sighted people on mobile devices. Our research question is: how do we help mobile users have a continuous reading experience while switching contexts?

In this paper, we present a user study with 10 mobile users designed to understand the differences between reading web pages under four different conditions: visual-reading, audio-listening, manual-switching between visual and audio, and auto-switching between them. The web pages are read with frequent context switching, i.e. from sitting to walking or from walking to sitting. The results showed that auto-switching not only provides significantly fewer missteps while walking than visual-reading, but it also provides the best reading experience.

Read4Me Browser is a prototype system we have built to support the presented study, including the four tested conditions. However, because of space limitation, we don't introduce Read4Me browser here.

\section{Methodology}

\section{Subjects}

Ten participants volunteered to join the study, 8 males and 2 females, on average 26.6 years old $(S D=2.37$ ). Study advertisements were sent out by email and posted as flyers on campus. Seven participants had not listened to audio books in the past, but all of them had used mobile phones and mobile web browsers. All participants are graduate students and all are fluent in English, although the first language of 7 out of 10 participants is not English.

\section{Experimental Design}

The goal of the study is to understand the differences between different approaches to reading on a mobile device. The approaches studied include visual reading, audio listening and the combination of both, all while reading content on a mobile device. We use web pages on a mobile web browser in particular, but the notion of the study could be extended to content reading in general on a mobile device.

The design of the user study came from existing literature [1][2][7] that focused on measuring human performance in mobile reading situations. Specifically, the study is modeled after the study by Vadas et al. [7] which examined overall performance and perceived workload for four conditions: audio-walking, audiositting, visual-walking, and visual-sitting. However, there are several differences between our study and theirs, including different examined conditions, periodic switching between sitting and walking, etc. 


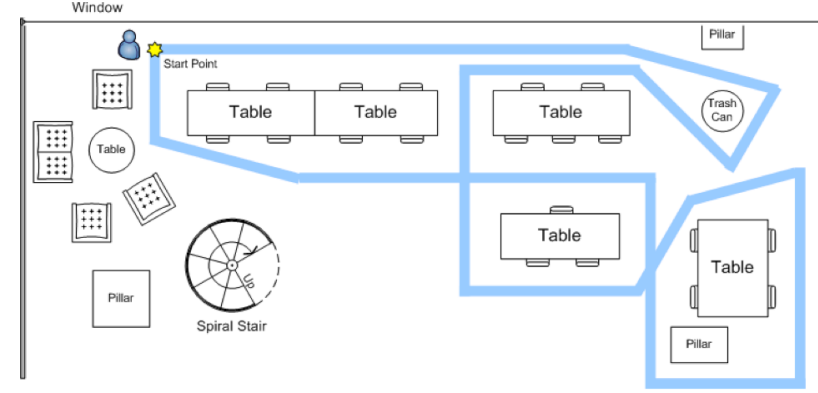

Figure 1 . The floor plan of user study experiment. The blue line is the path that participants had to follow.

\section{Procedure}

The user study setting is illustrated in Figure 1 . The path is taped on the ground and is $30 \mathrm{~cm}$ wide and 42.5 meters long. All participants are instructed to walk inside the taped path, and we recorded the number of out-ofpath steps during the study.

Four web pages were used in the user study: two articles selected from technology websites and another two from business websites. On average, there are 922.5 words per web page $(S D=82.5)$ and the study web pages are a subset of study materials from [8]. The web pages are formatted using mobile templates from the original web sites. The order of web pages presented is randomly selected, as well as the condition applied to those pages.

At the beginning of the study, the experimenter presented one sample web page and allowed the participant to get used to reading it in the four tested conditions. During the study, the experimenter raised a big sign and rang a bell to inform participants when to switch activities, e.g., from walking to sitting or vice versa. The switch happened once a minute to simulate frequent context switches happening on today's mobile usage [6]. The number of switches varied depending on the participant's reading time. For all conditions, all participants wore a wired headset and were asked to hold the phone as they walked through the path. On average, each study lasted for 70 minutes (SD = 12.71)
After each web page reading, the experimenter presented a questionnaire containing 5 multiple choice comprehension questions about the article and 2 subjective questions about ease of reading the web page and self-evaluation of understanding. At the end of each study, the experimenter conducted a post-test interview to solicit participants' subjective feedback.

There are four conditions investigated in the study: (1) visual-reading: participant reads the content visually both while sitting and while walking; (2) audio-

listening: participant listens to the content in both the sitting and walking states; (3) manual-switching: user manually switches to visual-reading or audio-listening for sitting or walking respectively; and (4) autoswitching: the mobile device automatically switches to visual-reading or audio-listening for sitting or walking respectively, using the orientation of the device to determine whether the user is carrying it in their hand for visual reading, or carrying it in a pocket for audio listening.

Each user experienced all four conditions, reading one article in each condition. The order of reading articles and applied methods were randomized. Although the experimenter informed participants to step only inside the taped path at the beginning of the study, the experimenter didn't remind participants again during the study. The experimenter kept track of this value as the participant walked, counting a step as out-of-path if at least half of the foot area was outside the blue taped path.

In the audio-listening, manual-switching and autoswitching conditions, we highlight the current listening sentence in red and the current listening paragraph with a yellow background (Figure 2). The listening paragraphs (up) with plain ones (down). 


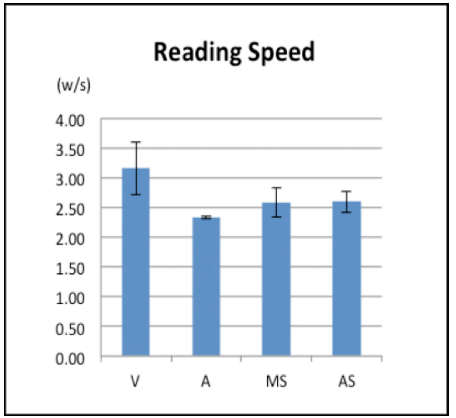

Figure 3. Reading speed for each condition, in words per second. Error bars show standard error.

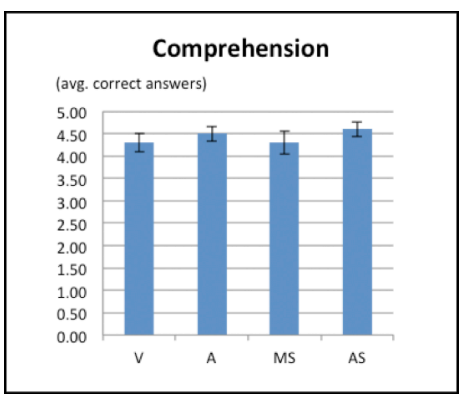

Figure 4. Comprehension for each condition (measured by number of correct answers to a 5-question quiz).

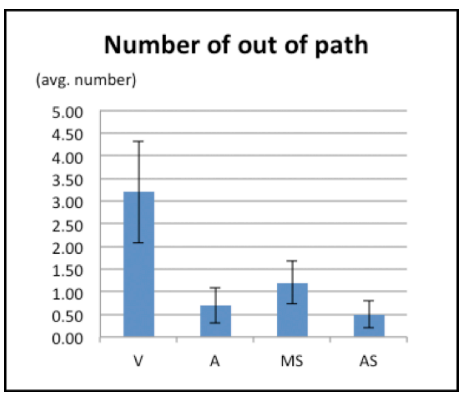

Figure 5. The number of out of path steps for each condition. paragraph is always scrolled automatically to the beginning of the screen. The main difference between manual-switching and auto-switching is whether one needs to manually press a button to start or stop listening to the web page.

In this user study, we measure: (1) reading speed; (2) reading comprehension; (3) number of out of path steps; (4) user satisfaction in ease of reading; and (5) self-evaluation of understanding. Reading speed means the number of words a participant reads per second, and reading comprehension is measured by the number of correct answers to the 5-question quiz. The user ratings of two subjective questions are used to measure user satisfaction in ease of reading and self-evaluation of understanding.

\section{Hypotheses}

In this user study, we had the following hypotheses we hoped to evaluate:

(H1) Visual-reading provides the best reading comprehension, but it also has the highest out-of-path rate.

( $\mathrm{H} 2)$ Audio-listening provides the best user satisfaction because it allows users to pay attention to surrounding environment and does not require extra burden for reading, such as visual attention.

H3) Auto-switching has lower reading comprehension than visual-reading, but more user satisfaction than visual-reading and less user satisfaction than audiolistening.

(H4) Manual-switching provides a similar reading comprehension as auto-switching, but it has a lower user satisfaction compared with auto-switching.

\section{Results}

We measured five variables in the study: reading speed, reading comprehension, number of out-of-path steps, user satisfaction of ease of reading and user's self-evaluation of understanding. It is a within-subject study and we analyzed the results using a one-way ANOVA. For easy explanation, we use $V, A, M S$ and $A S$ to represent visual-reading, audio-listening, manualswitching and auto-switching.

Figure 3 presents the average reading speed for each of the four conditions, measured in words per second. Although there is no clear overlap between the $V$ and $A$ error bars, statistically there is no significant difference across all 4 conditions. $\left(F_{3,36}=1.67, \mathrm{p}=0.19\right)$ The reason for the tiny variance in $A$, audio-listening, is because participants listened to spoken sentences generated using Text-to-Speech (TTS) at a constant speed that couldn't be changed during the study by participants.

Figure 4 shows the results of the reading comprehension measurement, which is defined as the number of correct answers out of 5 comprehension questions. There is no significant difference found in this measurement. $\left(F_{3,36}=0.54\right)$

Figure 5 presents the number of participants' steps out of the taped path in each condition. The measurement of the number of steps out of path is an indicator to understand participants' awareness of the surrounding environment. There is a significant difference in this measurement. $\left(F_{3,36}=3.53, p<0.05\right)$ A post-hoc Tukey test reports an honest significant difference between $V$ and $A S$ on the number of out-of-path steps $(\mathrm{q}=4.10, \mathrm{p}<0.05)$ and also gives an honest 


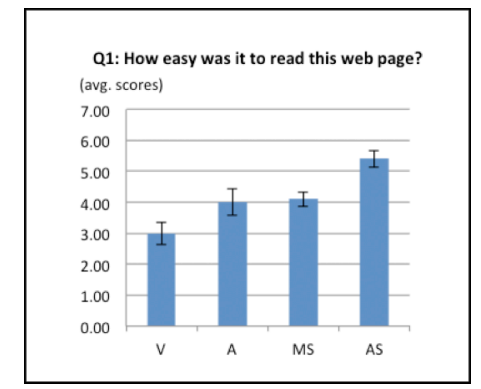

Figure 6. Ease of reading (rated on a scale from $1=$ very difficult to $7=$ very easy).

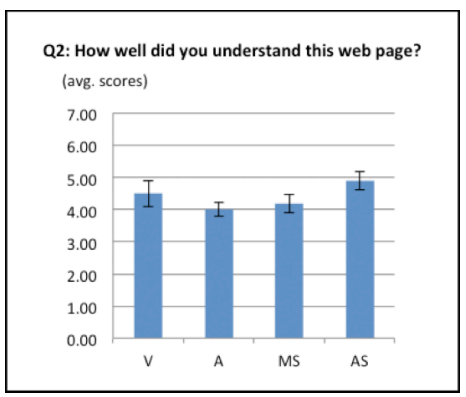

Figure 7. User's self-evaluation of understanding ( $1=$ very difficult, $7=$ very easy). significant difference between $V$ and $A(q=3.80, \mathrm{p}<$ 0.05).

Figure 6 shows subjective reading ease of the web page, on a Likert-type scale ( $1=$ very difficult, $7=$ very easy). There is a significant difference in subjective reading ease $\left(F_{3,36}=8.88, p<0.001\right)$. A post-hoc Tukey test gives an honest significant difference between $V$ and $A S(\mathrm{q}=7.26, \mathrm{p}<0.01), A$ and $A S(\mathrm{q}=4.24, \mathrm{p}<0.05)$, and $M S$ and $A S(\mathrm{q}=$ $3.93, \mathrm{p}<0.05)$. The result indicates that autoswitching provides the best reading ease of the web page among all four conditions.

Figure 7 reports on the second subjective question, self-evaluation of understanding, which is also a Likerttype scale question. There is no significant difference in self-evaluation of understanding. $\left(F_{3,36}=1.67, p=\right.$ 0.19)

Based on the results from the user study, we can address the hypotheses presented:

(1) Under the condition of frequent context switching between walking and sitting and requiring participants' attention for reading on a mobile device, visual-reading has a similar reading comprehension as audio-listening, manual-switching in between and auto-switching in between. However, visual-reading has the highest rate of stepping out of path, which is an indicator showing that participants might be more likely to encounter dangers when using visual-reading as a reading practice on a mobile device.

(2) Audio-listening does not provide the best user satisfaction. Instead, auto-switching, i.e. automatically switching between audio listening and visual reading on a mobile browser, provides the best user satisfaction.
(3) Auto-switching not only offers a similar reading comprehension as visual-reading, but it also provides the best user satisfaction among all four conditions. In fact, there are significant differences between using auto-switching and visual-reading in both number of steps out of path and the subjective ease of reading measurements. (Figure $6 \& 7$ )

(4) Manual-switching provides a similar reading comprehension as auto-switching, but there is no significant difference in user satisfaction between them.

\section{Limitations}

This study did not examine user performance during the context switch itself, such as from walking to sitting and from sitting to walking for each condition. Users might get lost during the transition and the performance might change accordingly, but the data presented only shows the user's performance with respect to the entire period of reading process. A further study should be conducted to understand this part.

\section{Discussion and Conclusion}

Mobile reading and browsing have many limitations originating from physical constraints. Our research attempts to enhance mobile reading by supporting continuous reading with frequent context switches, such as switching from sitting to walking or from walking to driving or hands-free situations. The main challenge in this scenario is that context switching will interrupt the reading process and the user might not understand where to start again, and may well just give up. 
In this paper, we address this problem and present the results of a user study modeled after the study by Vadas et al. [7] Results from our study include:

(1) All of four evaluated conditions, visual-reading, audio-listening, manual-switching and auto-switching, share a similar reading comprehension. However, visual-reading is more likely to expose readers to dangers than other conditions.

(2) Auto-switching not only has the lowest incidence of stepping out of path, which is safer for mobile reading, but it also has the best subjective ease of reading in the study, which means it provides the best reading experience when reading content on a mobile web browser under frequent context switching situation.

(3) Manual-switching provides a similar reading comprehension as auto-switching, and some study participants expressed the necessity of having this feature for certain conditions, such as listening to content while sitting in an office.

\section{Acknowledgements}

This research is conducted at MIT CSAIL. I appreciate my advisor Prof. Robert C. Miller's support and advice and the input from Hung-An Chang, Max Goldman and MIT UID group members. This work is supported in part by Quanta Computer as part of the TParty project.

\section{References}

1] Barnard et al. An empirical comparison of use-inmotion evaluation scenarios for mobile computing devices. International Journal of Human-Computer Studies, 62(4):487-520, 2005.

[2] Barnard et al. Capturing the effects of context on human performance in mobile computing systems. Personal and Ubiquitous Computing 11, 2, pp. 8196, 2006.

[3] Bigham, J., Prince, C. and Ladner, R. Webanywhere: A Screenreader On-the-go. W4A '08, 2008.

[4] Carter, S. and L. Denoue. SeeReader: An (Almost) Eyes-Free Mobile Rich Document Viewer. International Journal of Computer Science Issues (IJCSI), Vol. 1, pp. 36-41, 2009

[5] Gaal, A. et. al. Towards an Open Source Screen Reader: Screenreader Usability Extensions (SUE). In Proceedings of ICCHP, 2008.

[6] Oulasvirta, A. et al. Interaction in 4-second bursts The fragmented nature of attentional resources in mobile HCI. In Proceedings of CHI2005, ACM Press, pp. 919-928, 2005

[7] Vadas, K., Patel, N., Lyons, K., Starner, T. and Jacko, J. Reading on-the-go: a comparison of audio and hand-held displays. In MobileHCI, 2006.

[8] $\mathrm{Yu}, \mathrm{C} . \mathrm{H}$. and Miller, R.C. Enhancing web page readability for non-native readers. In Proceedings of CHI2010, ACM Press, 2010 